\title{
SIMULTANEOUS MULTI-WAVELENGTH OBSERVATIONS OF MICROQUASARS (THE MINE COLLABORATION)
}

\author{
Y. Fuchs ${ }^{1}$ J. Rodriguez, ${ }^{1,2}$ I. F. Mirabel, ${ }^{1}$ S. E. Shaw ${ }^{3,2}$ P. Kretschmar, ${ }^{4,2}$ M. Ribó, ${ }^{1}$ S. Chaty, ${ }^{5,1}$ \\ V. Dhawan, ${ }^{6}$ I. Brown,${ }^{7}$ R. Spencer, ${ }^{7}$ G. G. Pooley, ${ }^{8}$ and D. C. Hannikainen ${ }^{9}$
}

\begin{abstract}
RESUMEN
Presentamos la colaboración internacional MINE (Multi- $\lambda$ INTEGRAL NEtwork) cuya meta es llevar a cabo observaciones en múltiples longitudes de onda de microcuasares de forma simultánea al satélite INTEGRAL. Los primeros resultados con GRS 1915+105 son muy esperanzadores y junto con los que se obtendrán en el futuro nos ayudarán a comprender la física de los fenómenos de acreción y eyección relacionados con los sistemas binarios compactos.
\end{abstract}

\section{ABSTRACT}

We present the international collaboration MINE (Multi- $\lambda$ INTEGRAL NEtwork) aimed at conducting multiwavelength observations of microquasars simultaneously with the INTEGRAL satellite. The first results on GRS 1915+105 are encouraging and those to come should help us to understand the physics of the accretion and ejection phenomena around a compact object.

\section{Key Words: STARS: INDIVIDUAL (GRS 1915+105) — X-RAYS: BINARIES}

\section{INTRODUCTION}

Microquasars are X-ray binaries producing relativistic jets and thus appear as miniature replicas of distant quasars (Mirabel \& Rodríguez 1999). Their emission spectra, variable with time, range from the radio to the $\gamma$-ray wavelengths. We present here the first multi-wavelength campaign on GRS $1915+105$ involving the INTEGRAL satellite $(3 \mathrm{keV}-10 \mathrm{MeV})$. This campaign was conducted by the MINE (Multi- $\lambda$ INTEGRAL NEtwork, see http://elbereth.obspm.fr/ fuchs/mine.html) international collaboration aimed at performing multiwavelength observations of galactic X-ray binaries simultaneously with INTEGRAL.

\section{GRS $1915+105$}

The microquasar GRS $1915+105$ is extremely variable at all wavelengths (see Fuchs et al. 2003a for a review). It hosts the most massive known stellar mass black hole of our Galaxy with $M=14 \pm 4 M_{\odot}$ (Greiner et al. 2001). In the radio it can show superluminal ejections at arcsec scales (Nirabel \&

\footnotetext{
${ }^{1}$ Service d'Astrophysique, CEA/Saclay, France.

${ }^{2}$ INTEGRAL Science Data Center, Versoix, Switzerland.

${ }^{3}$ Cniversity of Southampton, Southampton, $\mathrm{CK}$.

${ }^{4}$ MPE, Garching, Germany.

${ }^{5}$ Université Paris 7, Paris, France

${ }^{6}$ National Radio Astronomy Observatory, Socorro, USA.

${ }^{7}$ Jodrell Bank, Manchester Univ., LK

${ }^{8}$ Mullard Radio Astronomy Obs., Cambridge, UK

${ }^{9}$ University of Helsinki, Helsinki, Finland.
}

Rodríguez 1994) leading to a maximum distance of $11.2 \pm 0.8 \mathrm{kpc}$ (Fender et al. 1999), and a compact jet at milli-arcsecond scales (Dhawan et al. 2000).

We conducted a multi-wavelength observation campaign of GRS $1915+105$ in spring 2003 (see Figure 1). Here we focus only on the April ubservations when ToO (Targets of Opportunity) were triggered by the MINE collaboration. This (nearly) simultaneous campaign involved the VLA, the VLBA, MERLIN and the Ryle Telescope (RT) in radio, the $\mathrm{ESO} / \mathrm{NTT}$ in IR, RXTE and INTEGRAL in $\mathrm{X} / \gamma$ rays. More details and description of the April 2 observations can be found in Fuchs et al. (2003b).
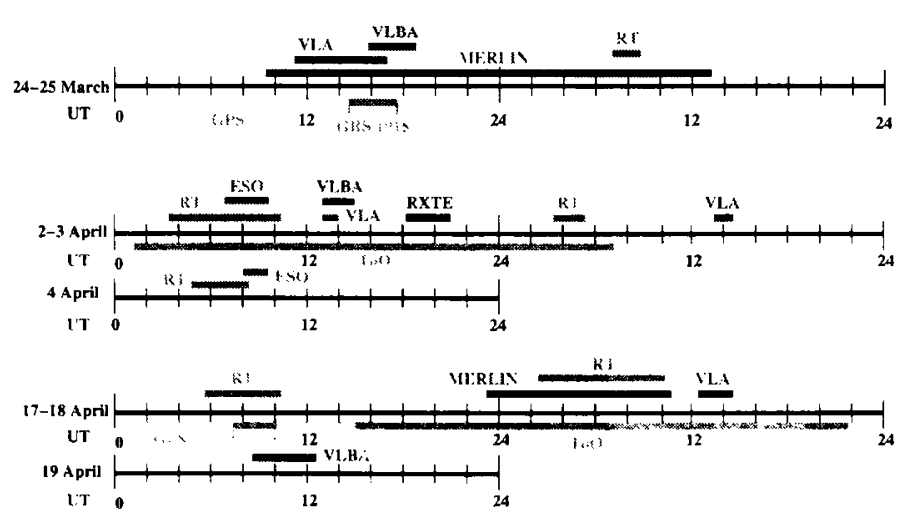

Fig. 1. Viewgraph of the observing campaign in spring 2003 , indicating the dates, time and involved observatories $($ GPS $=$ Galactic Plane Survey of INTEGRAL).

Our observations took place during the plateau state of GRS 1915+105 (Fender et al. 1999) i.e. quasi- 


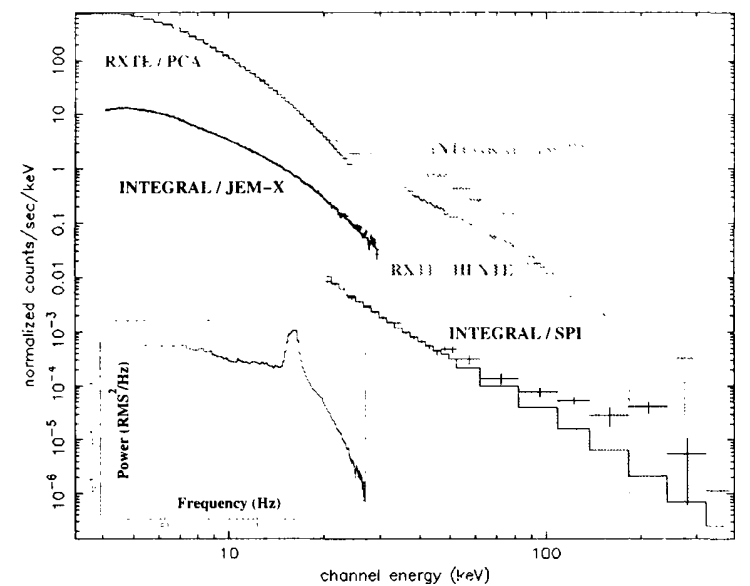

Fig. 2. $\mathrm{X} / \gamma$-ray spectra and fit of GRS $1915+105$ measured with RXTE \& INTEGRAL on April 2, 2003. The structures at $E>50 \mathrm{keV}$ in the SPI spectrum are instrumental background lines not adequately corrected. The PCA power density spectrum (inset) shows a clear QPO at $2.5 \mathrm{~Hz}$.
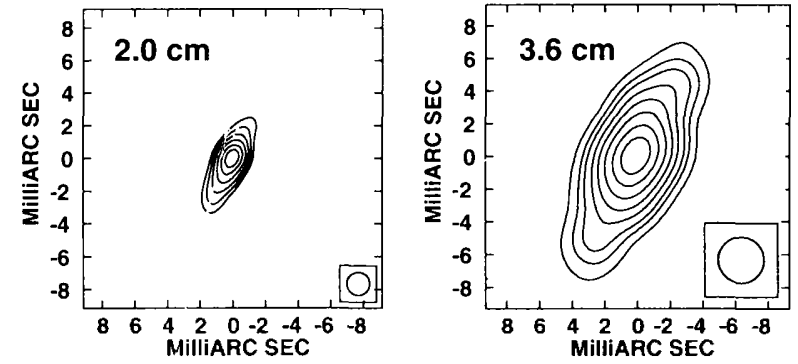

Fig. 3. VLBA images at $2.0 \& 3.6 \mathrm{~cm}$ on April 2, 2003 showing the compact, jet. 1 mas $=12 \mathrm{AU}$ at $12 \mathrm{kpc}$.

steady RXTE/ASM $(2-12 \mathrm{keV})$ flux $\sim 50 \mathrm{cts} / \mathrm{s}$ and high steady radio level ( $>100 \mathrm{mJy}$ ). On April 2 (Figure 2) \& 18 the high energy emission shows a power law dominated spectrum ( $>60 \%$ at $320 \mathrm{keV})$ with a photon index $\Gamma=2.9$ and 2.75 , respectively. This state is much softer than the classical low/hard state of the other $\mathrm{BH}$ binaries and is closer to the very high or intermediate states (McClintock \& Remillard 2004). The INTEGRAL observations show that this power law spectrum extends up to $400 \mathrm{keV}$ without any cutoff during this plateau state, consistent with the observations with CGRO/OSSE (Zdziarski et al. 2001). The estimated luminosity on April $2 \&$ 18 is respectively $\sim 7.5 \times 10^{38} \mathrm{erg} \mathrm{s}^{-1}$ overestimated using the PCA and $\sim 2.8 \times 10^{38} \mathrm{erg} \mathrm{s}^{-1}$ using JEM$\mathrm{X}$, corresponding to $40 \%$ and $16 \%$ of the Eddington luminosity for a $14 M_{\odot}$ black hole. As shown in Figure 2, a very clear Quasi-Periodic Oscillation (QPO) at $2.5 \mathrm{~Hz}$ with a $14 \% \mathrm{rms}$ level was observed in the $R X T E / \mathrm{PCA}$ signal, which is consistent with the previous observations of the plateau state (see also Rodriguez et al. 2003).

The VLBA high resolution images on April 2 (Figure 3) and 18 show the presence of a compact radio jet with a $\sim 7-14$ mas length $(85-170 \mathrm{AU}$ at $12 \mathrm{kpc}$ ). The optically thick synchrotron emission from this jet is responsible for the high radio levels. On April 18 the MERLIN image shows a radio extension $\left(\sim 0.3^{\prime \prime}\right)$ which is probably the trace of a superluminal ejection which occurred on April 4.

On April 2 the source was fairly bright in near-IR with an excess of $75-85 \%$ in the $K_{\mathrm{s}}$-band compared to the $K=14.5-15 \mathrm{mag}$. of the K-M giant donor star of the binary. According to the spectral energy distribution, this IR excess is compatible with a strong contribution from the synchrotron emission of the jet extending from the radio up to the near-IR. Different components, however, contribute to the IR in addition to the jet, such as the donor-star, the external part of the accretion disc or a free-free emission.

\section{CONCLUSIONS AND PROSPECTS}

Here for the first time, we observed simultaneously all the properties of the plateau state of GRS $1915+105$ : a powerful compact radio jet, responsible for the strong steady radio emission and probably for a significant part of the bright near-IR emission, as well as a QPO $(2.5 \mathrm{~Hz})$ in the $\mathrm{X}$-rays and a power law dominated $\mathrm{X}$-ray spectrum with a $\Gamma \sim 3$ photon index up to at least $400 \mathrm{keV}$. Forthcoming works will study detailed fits of the X-ray spectra, to determine for example whether this power law is due to an inverse Compton scattering of soft disc photons on the base of the compact jet or not. In order to better understand the unusual behaviour of GRS $1915+105$, we need to carry out similar simultaneous broad-band campaigns during the other states, in particular during the sudden changes that correspond to powerful relativistic ejection events.

\section{REFERENCES}

Dhawan, V., Mirabel, I. F., \& Rodríguez, L. F. 2000, ApJ, 543, 373

Fender, R. P., Garrington, S. T., McKay, D. J., et al. 1999, MNRAS, 304, 865

Fuchs, Y., Mirabel, I. F. \& Claret, A. 2003a, A\&A, 404, 1011

Fuchs, Y., Rodriguez, J., Mirabel, I. F., et al. 2003b, A\&A, 409, L35

Greiner, J., Cuby, J. G. \& McCaughrean, M. J. 2001. Nature, 414, 522

McClintock, J. E. \& Remillard, R. A. 2004, in 'Compact Stellar X-Ray Sources', eds. W.H.G. Lewin and M. van der Klis, CUP, astro-ph/0306213

Mirabel, I. F. \& Rodríguez, L. F. 1994, Nature, 371, 46 Mirabel, I. F. \& Rodríguez, L. F. 1999, ARA\&A. 37, 409 Rodriguez, J., Hannikainen D., et al. 2004, this volume Zdziarski, A. A., Grove, J. E., Poutanen, J., et al. 2001, ApJ, 554, L15 